\title{
Immunoexpression of IgA receptors (CD89, CD71) in dermatitis herpetiformis
}

\author{
Justyna Gornowicz-Porowska ${ }^{1}$, Agnieszka Seraszek-Jaros ${ }^{2}$, Monika Bowszyc-Dmochowska ${ }^{3}$, \\ Elzbieta Kaczmarek², Marian Dmochowski ${ }^{1}$
}

\author{
${ }^{1}$ Autoimmune Blistering Dermatoses Section, Department of Dermatology, Poznan University of \\ Medical Sciences, Poznan, Poland \\ ${ }^{2}$ Department of Bioinformatics and Computational Biology, Poznan University of Medical Sciences, \\ Poznan, Poland \\ ${ }^{3}$ Cutaneous Histopathology and Immunopathology Section, Department of Dermatology, Poznan \\ University of Medical Sciences, Poznan, Poland
}

\begin{abstract}
Introduction. The role of IgA receptors in dermatitis herpetiformis (DH) pathogenesis is still unknown. CD89 and CD71 may be associated with immune response during DH development. The purpose of this study was to perform semiquantitative analysis of simultaneous immunoexpression of CD89 and CD71 in DH and IgA/neutrophil-mediated non-DH dermatoses (IgAN) in relation to specific IgA autoantibodies/antibodies (tissue and epidermal transglutaminases, nonapeptides of gliadin - eTG/tTG/npG) as well neutrophil activation via the release of neutrophil elastase (NE).

Material and methods. In total, 48 patients were studied. The study was conducted on skin lesions and sera obtained from DH and IgAN patients. DH and IgAN served as mutually positive control groups. We used immunohistochemical technique with semiquantitative digital morphometry and ELISA to measure serum levels of anti-eTG/tTG/npG IgA.

Results. CD89 showed a significantly higher expression in DH than in IgAN. CD71 was overexpressed in DH and IgAN. CD89 immunoexpression correlated negatively with CD71 in IgAN. A positive correlation was revealed between CD89 immunoexpression and anti-npG IgA in DH. No statistically significant correlations were found in DH between the CD89/CD71 and NE immunoexpression, between CD71 immunoexpression and anti-tTG/ /eTG/npG IgA, or between CD89 immunoexpression and anti-eTG/tTG IgA serum levels.

Conclusions. CD89 is probably a key IgA Fc receptor in DH development, where it is associated with immune response to gluten. CD71 may be linked with inflammation in DH and IgAN. We suggest that interaction between CD89 and CD71 can modulate the inflammation in IgAN. (Folia Histochemica et Cytobiologica 2017, Vol. 55, No. 4, 212-220)
\end{abstract}

Key words: dermatitis herpetiformis; IgAN; Fc-alpha receptor; CD71; CD89; IHC

\section{Introduction}

Dermatitis herpetiformis (DH) belongs to IgA-mediated autoimmune blistering dermatoses. Its etiology

Correspondence address:

J. Gornowicz-Porowska, Ph.D., M.Sc.Eng.

Poznan University of Medical Sciences, Poznan, Poland

Autoimmune Blistering Dermatoses Section,

Department of Dermatology,

Przybyszewski 49, 60-355 Poznań, Poland

Tel.: 6186913 67, e-mail: justynagornowicz1@poczta.onet.pl is multifactorial and still equivocal with involvement of both genetic (HLA DQ2/DQ8 haplotypes) and environmental factors (gluten intake). Autoimmunity as well as inflammation/autoinflammation seem to be involved in DH progression [1] since a typical DH patient presents circulating IgA autoantibodies/antibodies against transglutaminases (mainly epidermal — eTG; tissue - tTG) and nonapeptides of gliadin (npG) coupled with rich neutrophilic infiltration within the dermal papillae. Activated neutrophils may release specific proteolytic enzyme (neutrophil elastase, NE) implicated in the destruction of 
dermal-epidermal junction (DEJ) with the cleavage within lamina lucida [2].

Direct immunofluorescence of perilesional skin revealed pronounced $\operatorname{IgA}$ deposits [3, 4], which may take various patterns (fine-granular, fibrillar, in dermal papillae, along the DEJ, a combination of all the above). These deposits are polyclonal, mainly composed of IgA1 [5].

Hendrix et al. [6] were the first to show the link between IgA deposition and recruitment of neutrophils in DH and $\mathrm{IgA} /$ neutrophil-mediated non-DH dermatoses (IgAN). This study also indicated immune adherence with $\mathrm{Fc}$ receptors engagement as the main mechanism of the proposed interaction [6].

Patients with DH have an associated gluten-sensitive enteropathy that is most often asymptomatic [7]. However, the development of skin eruption as well as cutaneous IgA deposits may be reduced by adhering to gluten-free diet.

IgAN are pathogenically closely related to $\mathrm{DH}$ sharing specific features of skin pathophysiology as they exhibit prominent infiltration of neutrophils and/or deposition of IgA. IgAN represent a heterogeneous group of autoimmune/inflammatory entities including linear IgA bullous dermatosis, IgA pemphigus, epidermolysis bullosa acquisita and subcorneal pustular dermatosis.

The formation of $\operatorname{IgA}$ (IgA1) deposits containing immunocomplexes forms a part of not yet fully elucidated DH pathogenesis. Although the factors that lead to the accumulation of neutrophils in the skin are not known, some key molecular factors contributing to the formation of immunocomplexes and neutrophilic microabscesses have been proposed. The involvement of human IgA Fc receptors, which may be associated with neutrophil activation, production of autoantibodies as well as gluten transport and/or transformation has been suggested in $\mathrm{DH}$. There are several known receptors for IgA in humans (e.g. polymeric Ig receptor, Fc-alpha/microR, CD89, CD71, mannose receptor) [8] differing in ligand preference and response to $\mathrm{Ig} \mathrm{A}$ binding.

CD89 (Fc-alphaRI) is a transmembrane glycoprotein expressed mainly on the surface of cells of the myeloid origin binding both IgA1 and IgA2 [9]. CD89 shows abundant expression on human neutrophils $[10,11]$ and mediates inflammatory responses to IgA-immunocomplexes [8]. CD71, the transferrin receptor (TfR), is ubiquitously expressed at low levels on normal cells and is expressed at greater levels on cells with a high proliferation rate such as cells of the basal epidermis and intestinal epithelium [12], binding preferentially to polymeric $\operatorname{IgA} 1[8,13]$. The data about the expression of transferrin receptor on neutrophils is contradictory; however, specific binding sites for $\mathrm{Tf}$ on the neutrophilic membranes was reported [14].

Some researchers showed that CD89 and CD71 were implicated in the pathogenesis of diseases with aberrant IgA synthesis and/or neutrophil tissue damage (IgA nephropathy, celiac disease - CD, rheumatoid arthritis, systemic lupus erythematosus), where functional abnormalities of CD89 and CD71 underlie their onset $[8,14-16]$. There are data indicating that the formation of IgA-CD89 may lead to stimulation of CD71 expression $[13,17]$. However, no attempts have been made to determine simultaneous expression of CD71 and CD89 in DH patients. Therefore, in the present study we have investigated the cutaneous immunoexpression of CD89 in concert with CD71 and their relationship with the expression of neutrophil elastase as well as levels of $\operatorname{IgA}$ autoantibodies/antibodies against eTG, tTG, npG in the peripheral blood of DH and IgAN patients.

\section{Material and methods}

This study was conducted after obtaining the local ethical committee approval (Poznan University of Medical Sciences, 953/14, Poznań, Poland, 2014).

Patients and sample collection. Altogether, clinical material from 48 patients with autoimmune blistering dermatoses before initiation of treatments was obtained. The examined material consisted of lesional skin tissues and sera from the examined groups. Samples were obtained from 33 patients with $\mathrm{DH}$ with active skin rash and $15 \mathrm{IgAN}$ patients as a positive control group pathogenetically closely related to $\mathrm{DH}$ due to 'neutrophilic endotype' and/or IgA autoantibodies production (involved 9 cases of linear IgA bullous dermatosis, 2 cases of $\operatorname{IgA}$ pemphigus, 3 cases of epidermolysis bullosa acquisita and 1 case of subcorneal pustular dermatosis). The DH and IgAN group served as mutual control groups.

To distinguish from $\mathrm{DH}$ and establish the diagnoses of IgAN, direct immunofluorescence and hematoxylin and eosin (H\&E) stainings were also done.

Patients were diagnosed and treated as well as samples were collected at the Department of Dermatology, Poznan University of Medical Sciences, Poland. The diagnosis of DH in all DH subjects was established according to: (i) positive DIF of perilesional skin (cutaneous IgA deposition in any of seven possible diagnostic patterns), (ii) histological features of DH (H\&E staining), (iii) detection of appropriate autoantibodies.

Paraffin-embedded skin tissue $4 \mu \mathrm{m}$-thick sections were mounted on poly-L-lysine coated glass slides. The serum used in the serological tests was taken at the time 


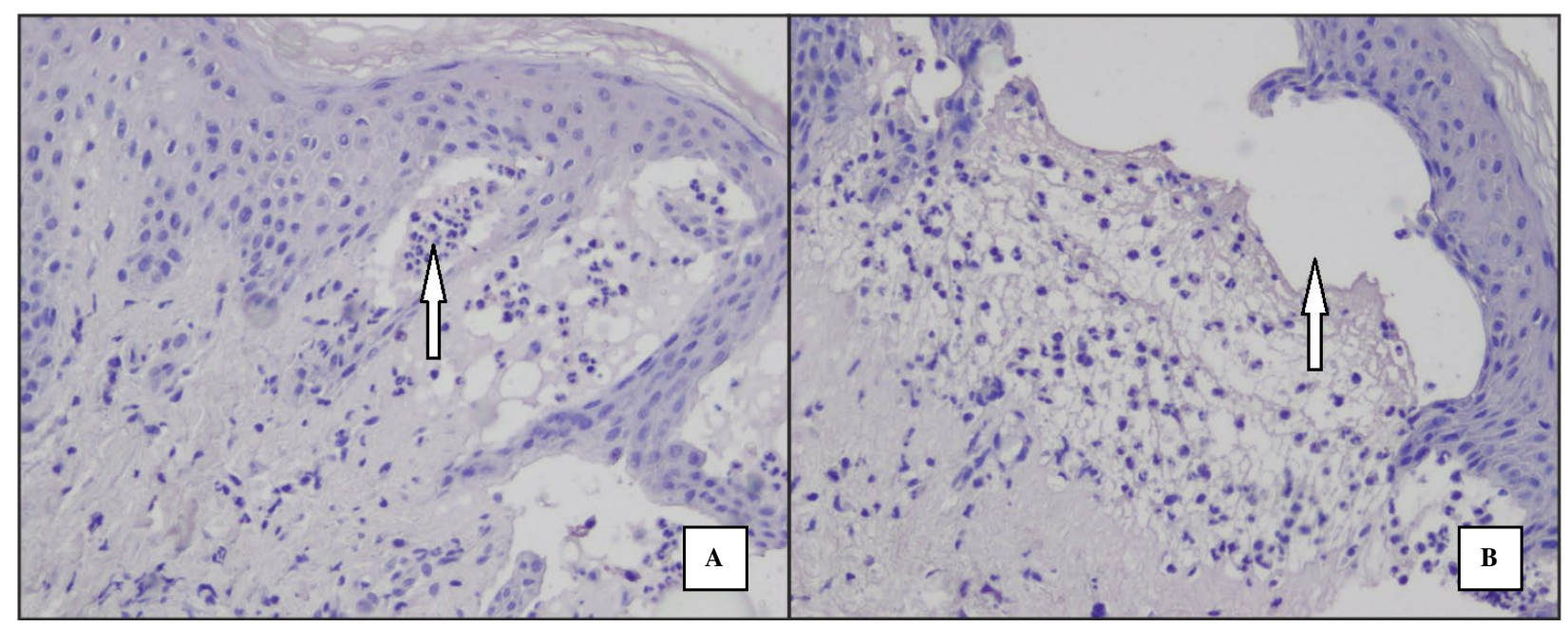

Figure 1. Negative immunocontrols by omission of the primary antibody (antibodies against either human CD89, CD71 or NE were replaced with PBS) for DH patient showing microabscesses of neutrophils at the tips of dermal papillae (arrow) with subepidermal cleaves above (A) and for IgAN (a patient with linear IgA bullous dermatosis) showing subepidermal blister (arrow) filled with neutrophils within and below the blister cavity (B). Abbreviations: DH — dermatitis herpetiformis; $\operatorname{Ig} \mathrm{AN}$ - IgA/neutrophil-mediated non-DH dermatoses. Original magnification $200 \times$.

of hospital admission/ambulatory care. Only patients, in whom both tissue sections of an adequate quality for processing and serum samples were available for investigation with IHC and ELISA, were subjected to correlation studies.

The NE expression was analyzed in the same patients as CD89 and CD71 expression. However, the group of patients with NE analysis was slightly greater because it was a subject of our previous analyses [18-20] which we broadened here with the detection of IgA Fc receptors.

Validation of IgA Fc receptors by immunohistochemistry. Immunohistochemical (IHC) staining with polyclonal rabbit antibodies against human CD89 (anti-CD89, Acris Antibodies GmbH, Herford, Germany), monoclonal murine antibodies against human CD71 (TFRC, Transferrin R antibody, Novus Biologicals, Littleton, CO, USA), monoclonal murine antibodies against human NE (clone NP57Dako, Glostrup, Denmark) and Real EnVision detection kit (Dako) were applied according to previously described procedures [19, 21]. All antibodies were diluted 1:100 in Antibody Diluent (Dako). The tests were conducted according to the classical Peroxidase-DAB staining as described in detail in our recent study [21].

IgA receptor staining was done after heat-induced epitope retrieval in an antigen retrieval solution, high $\mathrm{pH}$ (Dako). NE staining followed enzymatic digestion of sections with proteinase $\mathrm{K}$ (Dako).

Internal negative IHC procedure control reactions were based on substituting primary antibodies with phosphate-buffered saline (Fig. 1).

Evaluation of the results and microscopy image analysis. The slides were examined by light microscopy (BX40,
Olympus, Tokyo, Japan) connected to a digital camera and the images were recorded and archived. The images with positive IHC reaction were subjected to semiquantitative morphometric analysis using "HSV Filter" software originally developed in the Department of Bioinformatics and Computational Biology, Poznan University of Medical Sciences, Poland [21-23].

We measured the area of IHC reaction and calculated the staining intensity according to the following formula: (area of positive IHC reaction/area studied) $\times 100 \%$ [21-23]. Then, the mean value was calculated for every patient and each studied groups (DH, IgAN).

Immunoenzymatic assay. The concentrations of specific circulating serum autoantibodies were detected with commercially available ELISA tests. ELISAs were performed using the Euroimmun (Luebeck, Germany) kits to detect IgA autoantibodies/antibodies against (i) the fusion protein containing nonapeptides of gliadin (Antigliadin GAF-3X IgA ELISA), (ii) tTG (Anti-tTG IgA ELISA). The manufacturer-defined cut-off level was $25 \mathrm{RU} / \mathrm{ml}$ and $20 \mathrm{RU} / \mathrm{ml}$, respectively. The level of circulating serum IgA autoantibodies against eTG was detected with Anti-eTG ELISA (Immundiagnostik AG, Bensheim, Germany) with the manufacturer's cut-off value was $18 \mathrm{AU} / \mathrm{ml}$.

All measurements were made in ELISA plate reader (Expert 96, Asys Hitech GmbH, Eugendorf, Austria) equipped with Microwin 2000 software by a single operator following the manufacturer's instructions.

Statistical analysis. Significant differences in expression intensities of CD89/CD71/NE tissue expression were done using Wilcoxon signed rank test (comparison within examined group) and Mann-Whitney test (comparison 


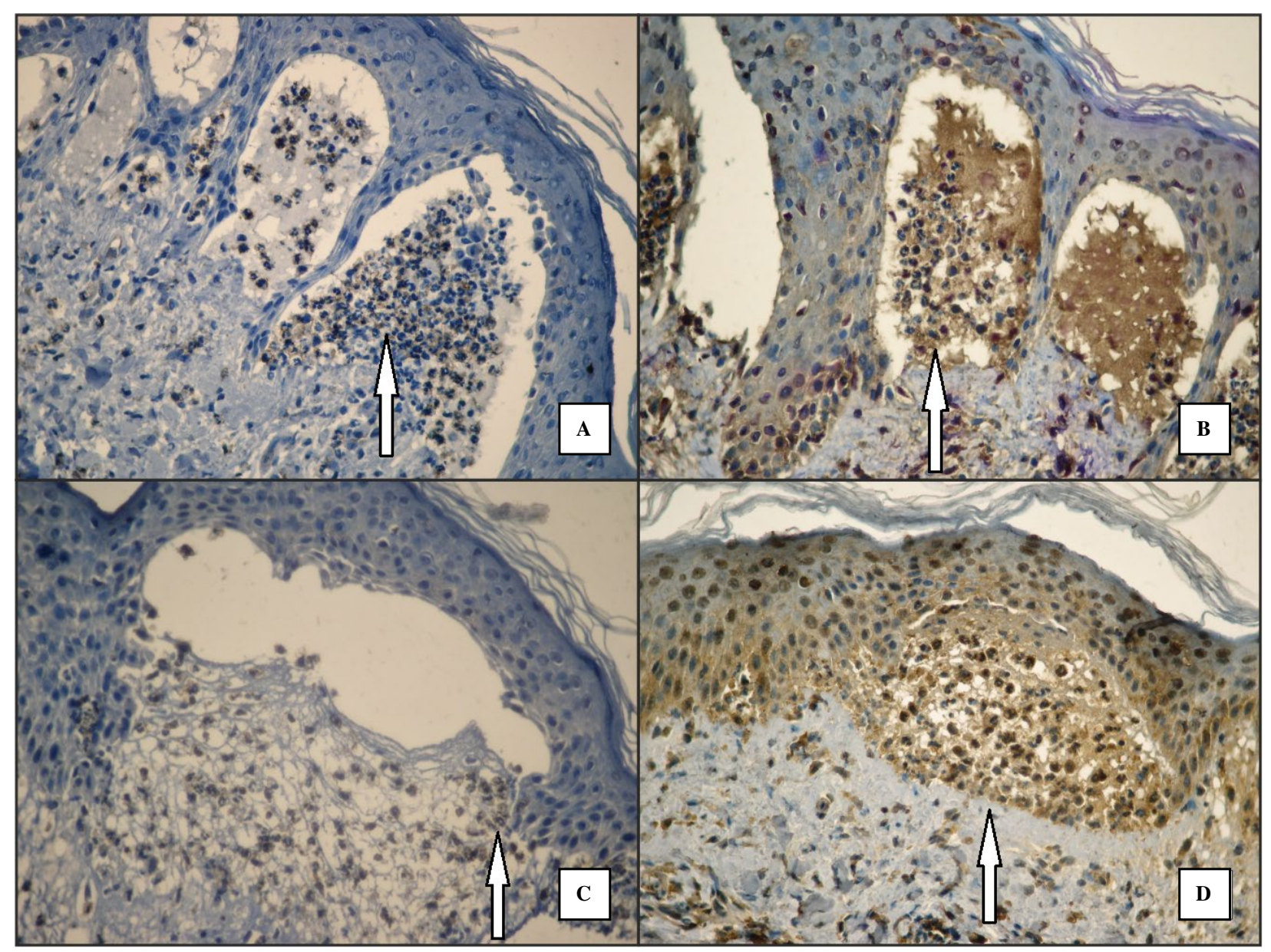

Figure 2. The cellular localization of CD89 (A, C) and CD71 $(\mathbf{B}, \mathbf{D})$ immunoreactivity in a representative DH patient (A and B) as well as IgAN patient (C and D). Areas showing the most intense immunoexpression are marked with arrows. Abbreviations as in the description of Figure 1. Original magnification $400 \times$.

between examined groups) with a significance level of $\mathrm{p}<$ $<0.05$. Correlations were calculated using Spearman's rank correlation coefficient. Statistical analyses were performed using statistical analysis software Statistica PL 10.0 (StatSoft, Cracow, Poland).

\section{Results}

\section{Immunoexpression of CD89 and CD71} in cutaneous lesions of $\mathrm{DH}$ and IgAN patients

The results of CD89 and CD71 expression analysis in representative $\mathrm{DH}$ and IgAN patients are shown on Figures 2, 3 and 4.

The tissue localization of CD89 in the DH involved mostly cell nucleus and membranes of neutrophils within microabscesses at papillary tips (Fig. 2A and 3A, B). CD89 expression in IgAN was poorly accented (Fig. 2C and 4A, B). CD71 immunoreactivity in DH and IgAN was observed frequently in the cytoplasm of neutrophils forming inflammatory infiltrates, however, the intensity of the reaction was irregular (Fig. 2B, D; 3C, D; 4C, D).

Semiquantitative results of CD89, CD71 and NE immunoexpression in the examined groups were presented in Table 1 . The intensity of cutaneous CD89 expression, detected in neutrophil-rich inflammatory infiltrates at the tips of dermal papillae in DH as well as within and below the blister cavity in linear IgA bullous dermatitis, was significantly increased in DH compared to IgAN ( $p=0.0432$, Mann-Whitney test). No difference in the cutaneous immunoexpression of CD71 between examined groups was found. The intensity of CD71 immunoreactivity was significantly higher as compared with CD89 both in DH ( $p=0.007$, Wilcoxon test) and $\operatorname{IgAN}(p=0.0008$, Wilcoxon test). Significantly lower NE than CD71 immunoexpression ( $p=0.0251$, Wilcoxon test) and slightly higher NE than CD89 immunoexpression ( $p=0.0469$, Wilcoxon test) were observed in DH. 


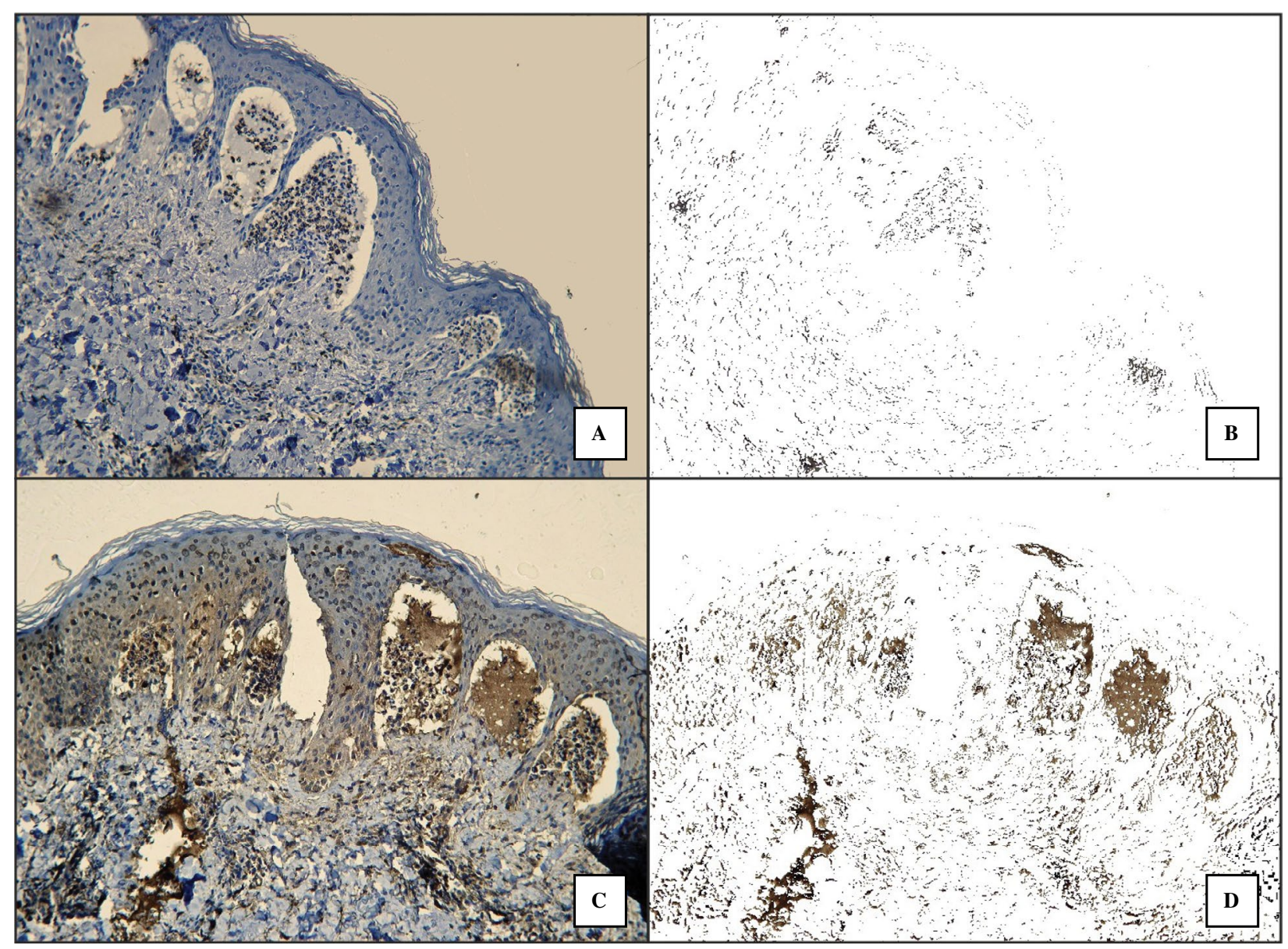

Figure 3. CD89 (A) and CD71 (C) immunoreactivity in lesional skin in a representative patient with DH who had serum level of anti-tTG IgA > $200 \mathrm{RU} / \mathrm{ml}$ as determined by ELISA. The intensity of CD89 (B) and CD71 (D) immunoreactivity was processed with digital microscopic image analysis as described in Methods. Abbreviations as in the description of Figure 1, tTG - tissue transglutaminase. Original magnification $200 \times$.

No correlation between cutaneous CD89/CD71 and NE expression was found in $\mathrm{DH}$. The moderate negative correlation between cutaneous CD89 immunoexpression and cutaneous CD71 immunoexpression in IgAN group $(r=-0.539$, Spearman's rank coefficient) was observed.

No immunoreactivity was found when control staining procedure was applied (Fig. 1).

\section{Evaluation of IgA FcRs in relation to the presence of autoantibodies/antibodies in serum}

Detailed analysis of anti-npG, anti-tTG, anti-eTG IgA levels obtained with ELISA tests in DH and IgAN is presented in Table 2. A positive relationship between CD89 expression intensities and the serum level of anti-npG IgA ( $r=0.664$, Spearman's rank coefficient) was revealed in DH. No correlations were observed between CD89 expression and anti-eTG, as well as anti-tTG IgAserum levels in DH. There was no correlation between the intensity of cutaneous CD71 expression and anti-npG, anti-tTG, anti-eTG IgA level in DH. All values of Spearman's rank coefficient between CD89/CD71 expression and IgA autoantibodies/antibodies levels in $\mathrm{DH}$ are presented in Table 3.

No correlations were observed between the intensity of cutaneous CD89/CD71 expression and anti-npG $(\mathrm{r}=0.173$ and $\mathrm{r}=0.018$, respectively), anti-tTG $(r=0.018$ in both cases) IgA level in IgAN.

\section{Discussion}

The major finding of the present study is that cutaneous immunoexpression of CD89 was significantly increased in patients with active DH compared with subjects with IgAN. Thus, it seems that CD89 may be associated with the remodeling of dermal-epidermal junction in DH. Nonetheless, additional multicenter study should be required to confirm the statistical data indicating the role of $\mathrm{Fc}$ receptors in $\mathrm{DH}$ with the use of larger number of untreated DH patients. It should 


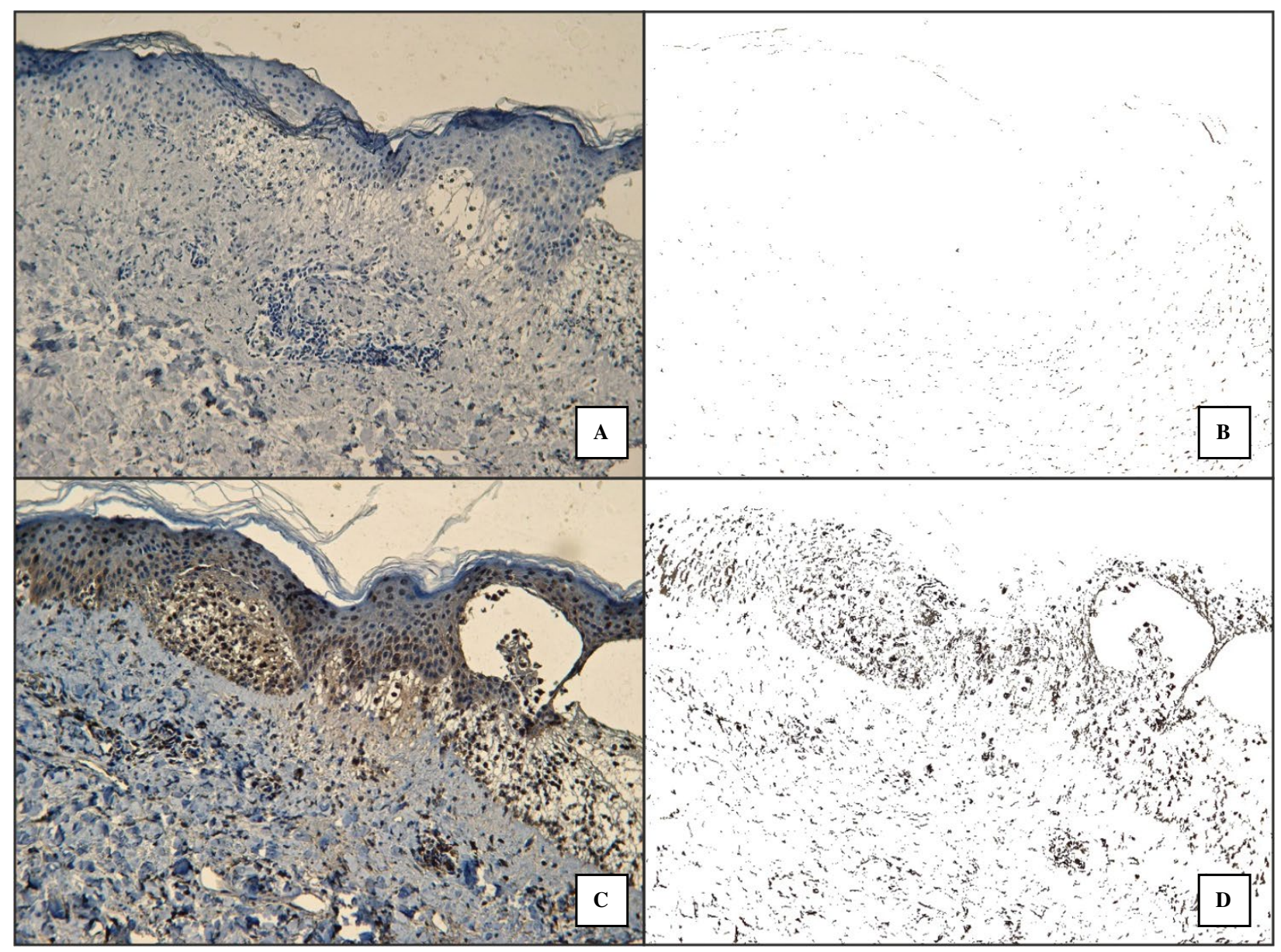

Figure 4. CD89 (A) and CD71 (C) deposits detected by immunohistochemistry in lesional skin in representative patients with IgAN. The intensity of CD89 (B) and CD71 (D) immunoreactivity was processed with digital microscopic image analysis as described in Methods. Abbreviations as in the description of Figure 1. Original magnification $200 \times$.

Table 1. Semiquantitative assessment of immunoexpression of CD89, CD71 and NE in dermatitis herpetiformis (DH), and CD89 and CD71 IgA/neutrophil-mediated non-DH dermatoses (IgAN)

\begin{tabular}{|l|c|c|c|c|c|c|}
\hline Variable & Group & Patients' number & Mean \pm SD (\%) & Median (\%) & Min. (\%) & Max. (\%) \\
\hline \multirow{2}{*}{ CD89 } & DH & 19 & $1.73 \pm 1.87$ & 1.19 & 0.00 & 8.81 \\
\cline { 2 - 7 } & IgAN & 15 & $0.87 \pm 0.51$ & 0.76 & 0.17 & 1.76 \\
\hline \multirow{2}{*}{ CD71 } & DH & 17 & $8.10 \pm 4.44$ & 6.17 & 3.03 & 19.72 \\
\cline { 2 - 7 } & IgAN & 15 & $12.78 \pm 9.45$ & 12.57 & 1.25 & 29.60 \\
\hline NE & DH & 24 & $3.46 \pm 2.31$ & 3.10 & 0.00 & 7.97 \\
\hline
\end{tabular}

Skin samples were stained by immunohistochemistry and the percentage of the area of immunoreaction in the analyzed skin lesions was determined as described in Methods. NS — statistically non-significant.

be noted that $\mathrm{DH}$ is a relatively rare skin disorder [24]. Smith et al. [7] found no difference in CD89 expression between active, ongoing $\mathrm{DH}$, inactive $\mathrm{DH}$ and normal subjects, whereas active DH patients showed enhanced function of CD89 compared with inactive $\mathrm{DH}$ and healthy population. It may be postulated that ligand binding to CD89 is regulated via inside-out signaling - thus cytokine stimulation of cells may modulate binding capacity in response to intracellular signals without affecting receptor expression levels [7]. It has been speculated that CD89 may become primed — as a result of cytokines' production — but surface receptor expression is not highly augmented [7]. Our results demonstrating no relationship between cutane- 
Table 2. Analysis of the results obtained with ELISA tests in dermatitis herpetiformis (DH) and IgA/neutrophil-mediated non-DH dermatoses (IgAN)

\begin{tabular}{|l|c|c|c|c|c|c|}
\hline Variable & Group & Patients'number & Mean \pm SD [U/ml] & Median [U/ml] & Min. [U/ml] & Max. [U/ml] \\
\hline \multirow{2}{*}{ Anti-npG IgA } & DH & 25 & $175.05 \pm 185.38$ & 107.55 & 6.67 & 644.11 \\
\cline { 2 - 7 } & IgAN & 11 & $3.25 \pm 6.24$ & 0.47 & 0 & 16.13 \\
\hline \multirow{2}{*}{$\begin{array}{l}\text { Anti-tTG } \\
\text { IgA }\end{array}$} & DH & 33 & $373.00 \pm 436.15$ & 200.00 & 4.08 & 1270.92 \\
\cline { 2 - 7 } & IgAN & 11 & $4.97 \pm 3.40$ & 3.99 & 1.39 & 11.48 \\
\hline Anti-eTG IgA & DH & 25 & $14.46 \pm 8.55$ & 13.85 & 2.37 & 34.02 \\
\hline
\end{tabular}

Abbreviations: eTG - epidermal transglutaminase; tTG — tissue transglutaminase; npG — nonapeptides of gliadin.

Table 3. Values of Spearman's rank coefficient between expression of CD89, CD71 (\% immunoreactive area per area of examined skin lesion) and levels of IgA autoantibodies/ /antibodies to eTG, tTG, npG in DH

\begin{tabular}{|l|l|l|}
\hline Parameter & CD89 & CD71 \\
\hline Anti-eTG IgA & 0.464 & 0.300 \\
\hline Anti-tTG IgA & 0.359 & 0.196 \\
\hline Anti-npG IgA & 0.664 & 0.217 \\
\hline
\end{tabular}

Abbreviations: eTG - epidermal transglutaminase; tTG — tissue transglutaminase; npG — nonapeptides of gliadin.

ous CD89 and NE expression are consistent with this thesis. Thus, possibly, CD89 is not directly involved in the activation of neutrophils through their locally enhanced expression in DH.

Our immunophenotypic characteristics of inflammatory cells within cutaneous eruption in $\mathrm{DH}$ and IgAN showed enhanced expression of CD71 in both groups of patients. However, the findings demonstrated different cellular localization of the IgA receptors as CD71 immunoreactivity was observed in the cytoplasm and CD89 immunoreactivity was associated with neutrophils. Previous data [25-28] indicated that CD71 is likely to be the most highly expressed as an early precursor with decreasing expression in maturing forms of the examined cells since strong and diffuse cytoplasmic staining for CD71, similarly as in neoplastic cells [28] and erythroid precursors [27], was reported. This may suggest that the overexpression of this protein concurs with the increased expression of its cognate receptor.

It has been suggested that $\operatorname{IgA} / \operatorname{IgA} 1$ binding to CD71 can circumvent the barrier effect of IgA and promote the entrance of immunogenic peptides into the subepithelial tissue. This effect depends on the upregulation of CD71 [29]. It has been also suggested that $\operatorname{IgA} / \operatorname{IgA} 1$ bound to $\mathrm{CD} 71$ may trigger the activation of local memory CD4 $+\mathrm{T}$ cells and thus perpetuate inflammation [30]. Therefore, it cannot be excluded that the overexpression of CD71, induced in various pathologies, such as anemia or intestinal infections [30], in conjunction with other unknown factors, might trigger the initial events inducing DH lesions in susceptible subjects. Heyman et al. [30] showed that protected transport of gliadin peptides occurs in active celiac disease patients via a CD71-mediated transcytosis of $\mathrm{IgA} /$ gliadin peptides immune complexes. Thus, perhaps, CD71 may be associated with gliadin transport in a similar way in $\mathrm{DH}$.

Interestingly, a significant positive correlation between cutaneous CD89 expression and anti-npG IgA was confirmed here, supporting our previous thesis that CD89 may be associated with gluten intolerance in DH rather than with enzyme-driven DEJ remodeling [20]. On the other hand, some researchers demonstrated the association between CD71 and tTG in CD and IgA nephropathy [31, 32], but our results do not confirm this interaction in DH. Our results about gliadin-CD89 relationship may be in line with certain data on IgA nephropathy. It is known that the chronic inflammation in patients with gluten intolerance may perpetuate the risk of kidney diseases [31]. Wijarnpreecha et al. [31] found that the risk of kidney diseases was significantly higher among patients with CD. It is postulated that gluten may exacerbate IgA nephropathy via gliadin-CD89 interaction. Data regarding CD71 and gluten hypersensitivity indicated that transferrin receptor is overexpressed in patients with $\mathrm{CD}$ [31], which was consistent with our findings in DH patients. CD89 seems not to be related with CD71 in DH development. Nonetheless, a negative relationship between CD89 and CD71 was found in IgAN. Thus, the inhibitory interaction of these receptors may be associated with the inflammation process in these dermatoses.

Our findings may help to understand the pathophysiological mechanism involving IgA Fc receptors related to the formation of neutrophilic microabscesses and $\operatorname{Ig} \mathrm{A} / \mathrm{Ig} \mathrm{A} 1$ deposition in $\mathrm{DH}$ suggesting CD89 as an important marker for $\mathrm{DH}$, where it is associated with immune response to gluten. 
Our work was limited by the relatively small number of DH patients. It was difficult to collect samples from patients with active and untreated DH for epidemiologic reasons and due to the monocenter nature of this study.

This study corroborates the findings of our previous work [19] indicating that there is no close relationship between the activation of neutrophils migrating to the skin and production of $\operatorname{IgA}$ autoantibodies to tTG/eTG sharing immunogenic epitopes in DH.

In conclusion, we suggest that CD89 seems to be a key receptor required for $\mathrm{DH}$ development acting as neutrophil function modulator. CD71 might be associated with inflammation both in DH and IgAN, probably due to the loss of protective function of IgA. CD71 and CD89 receptors can interact with each other to regulate the inflammation process in IgAN. CD89 seems to be related with gluten induced immune response in DH. Cutaneous CD71 expression is not linked with IgA anti-tTG/eTG/npG autoantibodies/antibodies production in $\mathrm{DH}$ as well as IgAN.

\section{Acknowledgments}

This study was funded by grant of the Polish Ministry of Science and Higher Education "Iuventus Plus" 0127/IPI/2015/73. A part of this study was presented at the Polish Academy of Dermatology and Venereology; XI Sympozjum Naukowo-Szkoleniowe PTD, Serock, 11-13 May 2017.

\section{References}

1. Gornowicz-Porowska J, Bowszyc-Dmochowska M, Seraszek-Jaros A, et al. Cutaneous expressions of interleukin-6 and neutrophil elastase as well as levels of serum IgA antibodies to gliadin nonapeptides, tissue transglutaminase and epidermal transglutaminase: implications for both autoimmunity and autoinflammation involvement in dermatitis herpetiformis. Cent Eur J Immunol. 2014; 39(3): 331-337, doi: 10.5114/ceji.2014.45944, indexed in Pubmed: 26155144.

2. Gornowicz-Porowska J, Bowszyc-Dmochowska M, Dmochowski M. Autoimmunity-driven enzymatic remodeling of the dermal-epidermal junction in bullous pemphigoid and dermatitis herpetiformis. Autoimmunity. 2012; 45(1): 71-80, doi: 10.3109/08916934.2011.606448, indexed in $\mathrm{Pu}-$ bmed: 21916544.

3. Zone JJ, Meyer LJ, Petersen MJ. Deposition of granular IgA relative to clinical lesions in dermatitis herpetiformis. Arch Dermatol. 1996; 132(8): 912-918, indexed in Pubmed: 8712841.

4. Kotze LM. Dermatitis herpetiformis, the celiac disease of the skin! Arq Gastroenterol. 2013; 50(3): 231-235, doi: 10.1590/ /S0004-28032013000200041, indexed in Pubmed: 24322197.

5. Volta U, Molinaro N, De Franchis R, et al. Correlation between IgA antiendomysial antibodies and subtotal villous atrophy in dermatitis herpetiformis. J Clin Gastroenterol. 1992; 14(4): 298-301, indexed in Pubmed: 1607605.

6. Hendrix JD, Mangum KL, Zone JJ, et al. Cutaneous IgA deposits in bullous diseases function as ligands to mediate adherence of activated neutrophils. J Invest Dermatol. 1990; 94(5): 667-672, indexed in Pubmed: 2182721.

7. Smith AD, Streilein RD, Hall RP. Neutrophil CD11b, L-selectin and Fc IgA receptors in patients with dermatitis herpetiformis. Br J Dermatol. 2002; 147(6): 1109-1117, indexed in Pubmed: 12452859.

8. Gomes MM, Herr AB. IgA and IgA-specific receptors in human disease: structural and functional insights into pathogenesis and therapeutic potential. Springer Semin Immunopathol. 2006; 28(4): 383-395, doi: 10.1007/s00281-006-0048-x, indexed in Pubmed: 17043868.

9. Moresco RN, Speeckaert MM, Zmonarski SC, et al. Urinary myeloid IgA Fc alpha receptor (CD89) and transglutaminase-2 as new biomarkers for active IgA nephropathy and henoch-Schönlein purpura nephritis. BBA Clin. 2016; 5: 79-84, doi: 10.1016/j.bbacli.2016.02.002, indexed in $\mathrm{Pu}-$ bmed: 27051593 .

10. Wehrli M, Cortinas-Elizondo F, Hlushchuk R, et al. Human IgA Fc Receptor Fc $\alpha$ RI (CD89) Triggers Different Forms of Neutrophil Death Depending on the Inflammatory Microenvironment. J Immunol. 2014; 193(11): 5649-5659, doi: 10.4049/jimmunol.1400028, indexed in Pubmed: 25339672.

11. Ben Mkaddem S, Rossato E, Heming N, et al. Anti-inflammatory role of the IgA Fc receptor (CD89): from autoimmunity to therapeutic perspectives. Autoimmun Rev. 2013; 12(6): 666-669, doi: 10.1016/j.autrev.2012.10.011, indexed in Pubmed: 23201915.

12. Daniels TR, Delgado T, Rodriguez JA, et al. The transferrin receptor part I: Biology and targeting with cytotoxic antibodies for the treatment of cancer. Clin Immunol. 2006; 121(2): 144-158, doi: 10.1016/j.clim.2006.06.010, indexed in Pubmed: 16904380.

13. Wiwanitkit V. IgA-CD89 complex in IgA nephropathy: a study on molecular function. Ren Fail. 2006; 28(6): 457-459, doi: 10.1080/08860220600767327, indexed in Pubmed: 16928613.

14. Maneva A, Taleva B. Receptors for transferrin on human neutrophils. Biotechnol \& Biotechnol Eq. 2009; 23: 477-479.

15. Monteiro RC. Role of IgA and IgA fc receptors in inflammation. J Clin Immunol. 2010; 30(1): 1-9, doi: 10.1007/s10875009-9338-0, indexed in Pubmed: 19834792.

16. Bairoch A, Apweiler R, Wu CH, et al. The Universal Protein Resource (UniProt). Nucleic Acids Res. 2005; 33(Database issue): D154-D159, doi: 10.1093/nar/gki070, indexed in Pubmed: 15608167.

17. Berthelot L, Papista C, Maciel TT, et al. Transglutaminase is essential for IgA nephropathy development acting through IgA receptors. J Exp Med. 2012; 209(4): 793-806, doi: 10.1084/ /jem.20112005, indexed in Pubmed: 22451718.

18. Gornowicz-Porowska J, Bowszyc-Dmochowska M, Seraszek-Jaros A, et al. Association between levels of IgA antibodies to tissue transglutaminase and gliadin-related nonapeptides in dermatitis herpetiformis. ScientificWorldJournal. 2012; 2012: 363296, doi: 10.1100/2012/363296, indexed in Pubmed: 22547981.

19. Gornowicz-Porowska J, Seraszek-Jaros A, Kaczmarek E, et al. IgA autoantibodies to gliadin nonapeptides, tissue transglutaminase and epidermal transglutaminase are associated, but unrelated to neutrophil elastase expression in lesional skin in human dermatitis herpetiformis. Postępy Dermatol Alergol. 2012; 4: 233-239, doi: 10.5114/pdia.2012.30461, indexed in Pubmed: 25339672.

20. Gornowicz-Porowska J. Expression of neutrophil elastase in relation to the autoimmunity to epidermal transglutaminase, BP180, BP230, desmoglein 1 and 3 in autoimmune blistering 
dermatoses. Saarbrücken, Germany: LAP Lambert Academic Publishing GmbH\&Co Kg. ; 2012.

21. Gornowicz-Porowska J, Seraszek-Jaros A, Bowszyc-Dmochowska M, et al. A comparative study of expression of Fc receptors in relation to the autoantibody-mediated immune response and neutrophil elastase expression in autoimmune blistering dermatoses. Pol J Pathol. 2017; 68(2): 109-116, doi: 10.5114/pjp.2017.69686, indexed in Pubmed: 29025244.

22. Kasprzak A, Szmyt M, Malkowski W, et al. Analysis of immunohistochemical expression of proinflammatory cytokines (IL-1 $\alpha$, IL-6, and TNF- $\alpha$ ) in gallbladder mucosa: comparative study in acute and chronic calculous cholecystitis. Folia Morphol (Warsz). 2015; 74(1): 65-72, doi: 10.5603/FM.2015.0011, indexed in Pubmed: 25792398.

23. Kasprzak A, Rogacki K, Adamek A, et al. Tissue expression of $\beta$-catenin and $\mathrm{E}$ - and $\mathrm{N}$-cadherins in chronic hepatitis $\mathrm{C}$ and hepatocellular carcinoma. Arch Med Sci. 2017; 13(6): 1269-1280, doi: 10.5114/aoms.2017.65272, indexed in $\mathrm{Pu}-$ bmed: 29181057.

24. Bakema JE, van Egmond M. The human immunoglobulin A Fc receptor $F_{c} \alpha$ RI: a multifaceted regulator of mucosal immunity. Mucosal Immunol. 2011; 4(6): 612-624, doi: 10.1038/ /mi.2011.36, indexed in Pubmed: 21937986.

25. Hall RP, Takeuchi F, Benbenisty KM, et al. Cutaneous endothelial cell activation in normal skin of patients with dermatitis herpetiformis associated with increased serum levels of IL-8, sE-Selectin, and TNF-alpha. J Invest Dermatol. 2006; 126(6): 1331-1337, doi: 10.1038/sj.jid.5700277, indexed in Pubmed: 16575390.

26. Müllner EW, Kühn LC. A stem-loop in the 3' untranslated region mediates iron-dependent regulation of transferrin receptor mRNA stability in the cytoplasm. Cell. 1988; 53(5): 815-825, indexed in Pubmed: 3370673.

27. Marsee DK, Pinkus GS, Yu H. CD71 (transferrin receptor): an effective marker for erythroid precursors in bone marrow biopsy specimens. Am J Clin Pathol. 2010; 134(3): 429-435, doi: 10.1309/AJCPCRK3MOAOJ6AT, indexed in Pubmed: 20716799.

28. Magro G, Cataldo I, Amico P, et al. Aberrant expression of TfR1/CD71 in thyroid carcinomas identifies a novel potential diagnostic marker and therapeutic target. Thyroid. 2011; 21(3): 267-277, doi: 10.1089/thy.2010.0173, indexed in Pubmed: 21323588.

29. Korneychuk N, Meresse B, Cerf-Bensussan N. Lessons from rodent models in celiac disease. Mucosal Immunol. 2015; 8(1): 18-28, doi: 10.1038/mi.2014.102, indexed in Pubmed: 25354320

30. Heyman M, Menard S. Pathways of gliadin transport in celiac disease. Ann N Y Acad Sci. 2009; 1165: 274-278, doi: 10.1111/j.1749-6632.2009.04032.x, indexed in Pubmed: 19538316.

31. Wijarnpreecha K, Thongprayoon C, Panjawatanan P, et al. Celiac disease and the risk of kidney diseases: A systematic review and meta-analysis. Dig Liver Dis. 2016; 48(12): 1418-1424, doi: 10.1016/j.dld.2016.08.115, indexed in Pubmed: 27633269 .

32. Lebreton $\mathrm{C}$, Ménard $\mathrm{S}$, Abed $\mathbf{J}$, et al. Interactions among secretory immunoglobulin A, CD71, and transglutaminase-2 affect permeability of intestinal epithelial cells to gliadin peptides. Gastroenterology. 2012; 143(3): 698-707.e4, doi: 10.1053/j.gastro.2012.05.051, indexed in Pubmed: 22750506.

Submitted: 21 May, 2017

Accepted after reviews: 23 January, 2018

Available as AoP: 5 February, 2018 